\title{
SCANNING-FIT ANALYSIS OF fs-SPECTROSCOPIC DATA
}

\author{
MARK BISCHOFF*, GREGOR STOBRAWA \\ and SABINE RENTSCH \\ Friedrich-Schiller-University Jena, Institute for Optics and Quantum \\ Electronics, Max-Wien-Platz 1, D-07743 Jena, Germany
}

(Received 27 March 2000)

\begin{abstract}
The scanning-fit method was developed for the global data analysis of pump-probe data. This method is useful in cases when pulse durations are adjusted to be short compared with nuclear dynamics and long compared with the dephasing timescales. Then multiexponential functions can be used to describe the mono molecular relaxation processes and the scanning-fit method increases the accuracy of the calculated time constants, yields detailed information about their ranges of confidence and their dependency from each other. In this paper we describe the method in detail and demonstrate its application to simulated data. Additionally, we report about the results which were obtained from scanning-fit analysis in case of the photoisomerization of pinacyanol and the light induced processes in phycocyanobilin.
\end{abstract}

Keywords: fs-spectroscopy; Data analysis; Photoisomerization

\section{INTRODUCTION}

The methods of nonlinear optical spectroscopy make it possible to study certain aspects of molecular processes in their characteristic timescales. Transient absorption spectroscopy is one of the most common experimental techniques within this field. It is based on the subjection of the sample to two light pulses: The pump and the probe pulse with wavelengths $\lambda_{0}$ and $\lambda_{1}$ and pulse length $\tau_{P}$ for both pump and probe pulse. The pump pulse interacts with the sample at time $t_{o}$ and the

*Corresponding author. 
probe pulse is delayed by time $t$ with respect to the pump pulse $\left(t>\tau_{P}\right)$. The signal is obtained from the energy of the transmitted probe pulse. From this signal the change of the absorption of the sample can be estimated as a function of the delay time $t$ and the wavelength $[1,2]$.

One of the most common goals of pump-probe experiments is to elucidate the population dynamics of electronically excited states. In case of mono molecular processes the transient absorption at selected wavelengths can be decomposed into a sum of exponentials.

$$
\Delta A_{\lambda_{0}, \lambda_{1}}(t)=\sum_{j} a_{i} \mathrm{e}^{-\left(t / \tau_{i}\right)}
$$

Different numerical data analysis methods can be used to find the best fit-coefficients of this function. Nevertheless, in cases with large numbers of these coefficients their estimation can be rather difficult. If the fit-function is of the type Eq. (1) this behaviour is connected with the high dependency of the coefficients $\tau_{i}$ on each other (correlation between the coefficients). Therefore the fit-analysis of experimental data obtained from pump-probe experiments is encumbered with problems. This is the case even if global fit-methods are applied to experimental data with a high signal-noise ratio $(S / N)$. In contrast to other fit methods, the result of our scanning-fit method is not only a point estimation of the fit-coefficients but a set of solutions. From its properties the range of confidence and the correlations of the fit-coefficients can be obtained. Our method can also be successfully applied in the case of non-exponential fit-functions [3].

\section{THE METHOD OF SCANNING-FIT}

\subsection{Principles of Fit-algorithms}

We consider the case that the obtained experimental data $\left\{y_{j}\right.$, $\left.\left\{x_{1 . . m}\right\}\right\},(j=1 . . a)$ can be described with a function $f\left(p,\left\{x_{1 . . m}\right\}\right)$. It is of great practical interest to find the best vector of fit-coefficients $\tilde{p}=\left(p_{1}, \ldots, p_{n}\right)$. The mathematical problem to solve is the estimation of the global minimum of the chi-squared function $F\left(p, y_{j}\right.$, $\left.\left\{x_{1 . . m}\right\}_{j}\right)$. This function is the standard error of difference between the 
experimental data and the fit-function $f\left(p,\left\{x_{1 . . m}\right\}\right)$.

$$
\begin{aligned}
& F\left(p, y_{j},\left\{x_{1 . . m}\right\}_{j}\right)=\frac{1}{a} \sum_{j=1}^{a}\left(f\left(p,\left\{x_{1 . . m}\right\}_{j}\right)-y_{j}\right)^{2} \\
& \forall p: \tilde{F}=F\left(\tilde{p}, y_{j},\left\{x_{1 . . m}\right\}_{j}\right) \leq F\left(p, y_{j},\left\{x_{1 . . m}\right\}_{j}\right)
\end{aligned}
$$

A number of sophisticated numerical methods exist which can be used to solve this problem. They should yield the point $\tilde{p}$ from the space of coefficients for which $F\left(\tilde{p}, y_{j},\left\{x_{1 . . m}\right\}_{j}\right)$ is minimal. Nevertheless, sometimes their results characterize only a local minimum but not the global minimum of $F$. Different problems can lead to this unfavourable result. (i) The most important one is the limited signal-noise ratio $(S / N)$ of experimental data. This results in a flattening of the function $F$. It follows from this behaviour that several local minima can exist making the estimation of the global minimum rather difficult. (ii) Additionally, conventional fit-algorithms always yield exactly one vector of coefficients $p$. This contradicts the fact that for $p$ many different solutions might exist having comparable probabilities. (iii) Alternative solutions are excluded and the solution arrived at depends on the start coefficients used.

To estimate the range of confidence of the solution found the dependencies of the $p_{i}$ on each other are to be taken into consideration. Normally, the range of confidence of the estimated coefficients is not symmetric, thus it is not an $n$-dimensional sphere.

\subsection{The Basics of the Scanning-fit}

In general the coefficients $p_{i}$ determine the shape of the function $f$ in different ways. The method of scanning-fit makes use of this property to estimate all solutions for $\tilde{p}$ which are suitable for describing experimental data which have a limited $S / N$. In this section we will discuss the principle of the scanning-fit method and its application more generally. Nevertheless, we limit the explanations to the case of one variable $x(m=1$ in Eq. (2)). The idea of the scanning-fit method can easily be understood if one looks at the example of a multi-exponential fit-function Eq. (1). (We write this fit-function as dependent on the variable $x=t$ to avoid confusion 
with general equations.)

$$
f\left(A_{i}, \tau_{i}, x\right)=\sum_{i} A_{i} \mathrm{e}^{-\left(x / \tau_{i}\right)}
$$

In practice, if conventional fit-algorithms are used for the data analysis they yield the amplitudes $A_{i}$ of Eq. (4) more accurately than the time constants $\tau_{i}$. The reasons for this are explained in the section above. The selection of the coefficients $\tau_{i}$ splits the space of the coefficients into the subspaces of the critical coefficients $p_{c}$ and the remaining coefficients $p_{r}$ ( $\tau_{i}$ and $A_{i}$ respectively). This kind of dividing of the space of coefficients is possible in general. Nevertheless, it depends on the mathematical structure of the fit function.

Following the splitting of the set of coefficients $P$ the function $F\left(p, y_{j}, x_{j}\right)$ can be understood as depending on the set of critical coefficients and all remaining coefficients $F\left(p_{c}, p_{r}, y_{j}, x_{j}\right)$. In the following sections the minimum of $F$ with the condition of fixed $p_{c}$ we name $F\left(p_{c}\right)$.

The principle of the introduced method involves (i) the splitting of the space of coefficients into two subspaces and (ii) the systematic scanning within the subspace of critical coefficients. For each set of critical coefficients chosen all remaining coefficients are to be optimized by any suitable conventional fit algorithm. The numerical result of this analysis method is a set of points of support for the function $F\left(p_{c}\right)$ within the scanned subspace of the critical coefficients.

In practice the base vectors for every critical coefficient $\left(p_{c}\right)_{i}$ are defined within its domain of definition. For every selected combination of $p_{c}$ all remaining coefficients $p_{r}$ are to be optimized with a suitable conventional fit-algorithm. In this way the values of the function $F$ can be estimated as a function of $p_{c}$. In the case of two critical coefficients, $F$ can be visualized as a surface over a two-dimensional plane. In case there are more than two critical coefficients two-dimensional cuts through the obtained hyper surface make it possible to understand the numerical result. In this way the method of scanning-fit yields all local minima of the function $F$ within the scanned range of coefficients. The set of solutions $\mathcal{L}_{\alpha}$ can be estimated if the level of significance $\alpha$ is given.

$$
\mathcal{L}_{\alpha}=\left\{p_{c}, \forall \alpha \geq 0: F\left(p_{c}\right) \leq(1+\alpha) \cdot F\left(\tilde{p}_{c}\right)\right\}
$$


where $F\left(\tilde{p}_{c}\right)$ is the minimum of $F\left(p_{c}\right)$ within the subspace of critical parameters. For the choice of a significance level the $S / N$ of the experimental data should be taken into consideration. In case of normally distributed noise $R(\sigma)$, with the variance $\sigma^{2}$, a useful criterion for $\alpha$ is

$$
\forall \xi \leq \alpha: \forall p_{c} \epsilon \mathcal{L}_{\xi}: \forall x_{j}:\left|f\left(p_{c}, x_{j}\right)-f\left(\tilde{p}_{c}, x_{j}\right)\right|<\sigma
$$

This criterion means that the destination between each fit-function and the data points is always smaller then $\sigma$.

In conclusion, the introduced method yields the set of solutions for the critical coefficient vector $p_{c}$. The shape of the set of solutions shows the correlation of the critical coefficients $\left(p_{c}\right)_{i}=\left(p_{c}\right)_{i}\left(\left(p_{c}\right)_{j \neq i}\right)$. Ranges of confidence for every component of $p_{c}$ can be estimated from the extension of the set of solutions. Nevertheless, these ranges depend on the choice of all other coefficients. The set of solutions do not have to be connected and its shape can be complicated. It is obvious that the graph of the set of solutions exceeds a point estimation combined with its range of confidence.

\subsection{Demonstration of the Scanning-fit with Simulated Data}

In this section we demonstrate the application of the Scanning-fit method to simulated data points [4]. To simulate different $S / N$ we have used the modified Eq. (4) with a normally distributed noise $R(\sigma)$, with the variance $\sigma^{2}$.

$$
y(t)=A^{\prime} \cdot \mathrm{e}^{-\left(t / \tau_{1}^{\prime}\right)}+\left(1-A^{\prime}\right) \cdot \mathrm{e}^{-\left(t / \tau_{2}^{\prime}\right)}+R(\sigma)
$$

The fit-function was

$$
f(t)=A \cdot \mathrm{e}^{-\left(t / \tau_{1}\right)}+(1-A) \cdot \mathrm{e}^{-\left(t / \tau_{2}\right)} .
$$

The coefficients $\tau_{i}$ were selected as the critical coefficients. The domain of these critical coefficients was scanned and the remaining coefficient, $A$, was again and again optimized using the fit-algorithm of Levenberg and Marquardt. Different results were obtained depending on the coefficients used for the simulation of the data $\left(\tau_{i}\right.$ and $\left.A\right)$. Their plots are given in Figure 3 as density plots (value of $F\left(\tau_{1}, \tau_{2}\right)$ is the blackness). White points represent functions with low chi-square between 


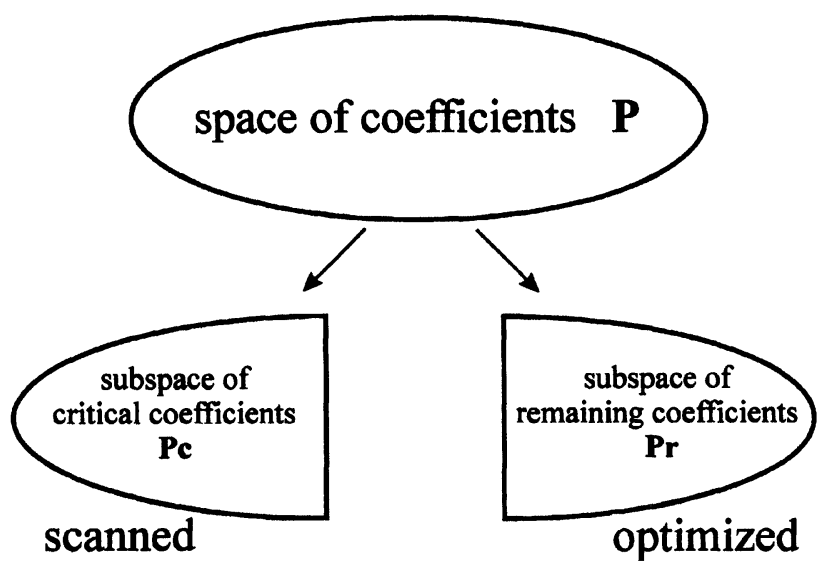

FIGURE 1 Separation of the coefficient space into subspaces of critical coefficients and remaining coefficients.

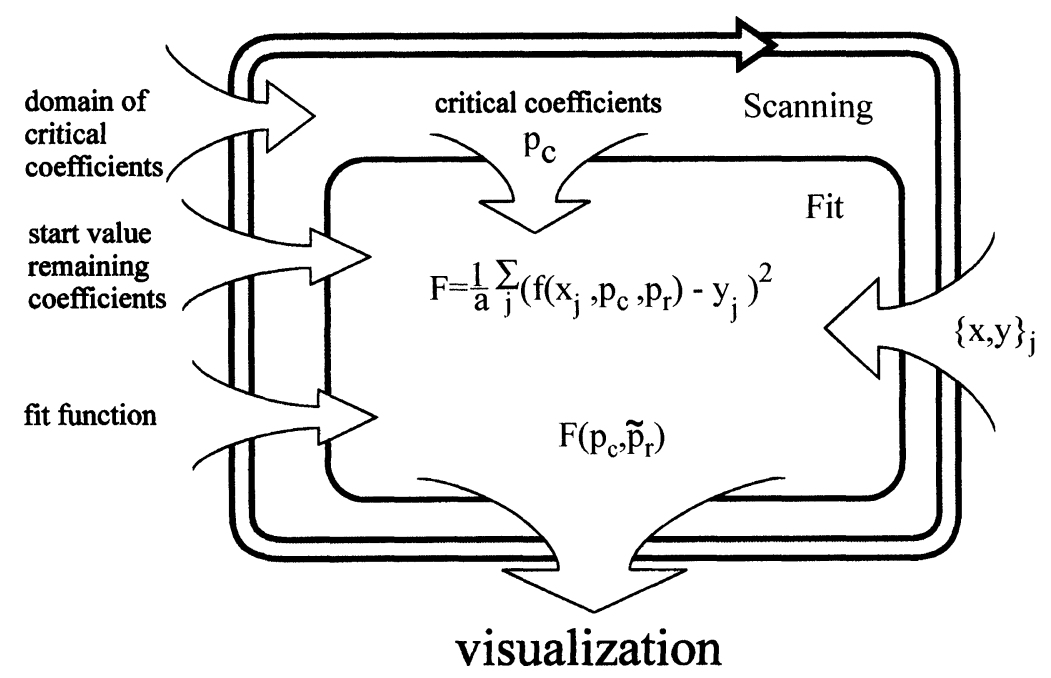

FIGURE 2 Scheme of the scanning-fit algorithm.

the simulated data points and the function (for details see figure subscription). The set of solutions $\mathcal{L}_{\alpha}$ is surrounded by a black curve in Figure 3. All fit-functions $f\left(p_{c} \in \mathcal{L}_{\alpha}, x_{j}\right)$ satisfy the condition Eq. (6).

The graphs in Figure 3 show some common properties. The interchangeability of the two exponentials causes a symmetry with respect 


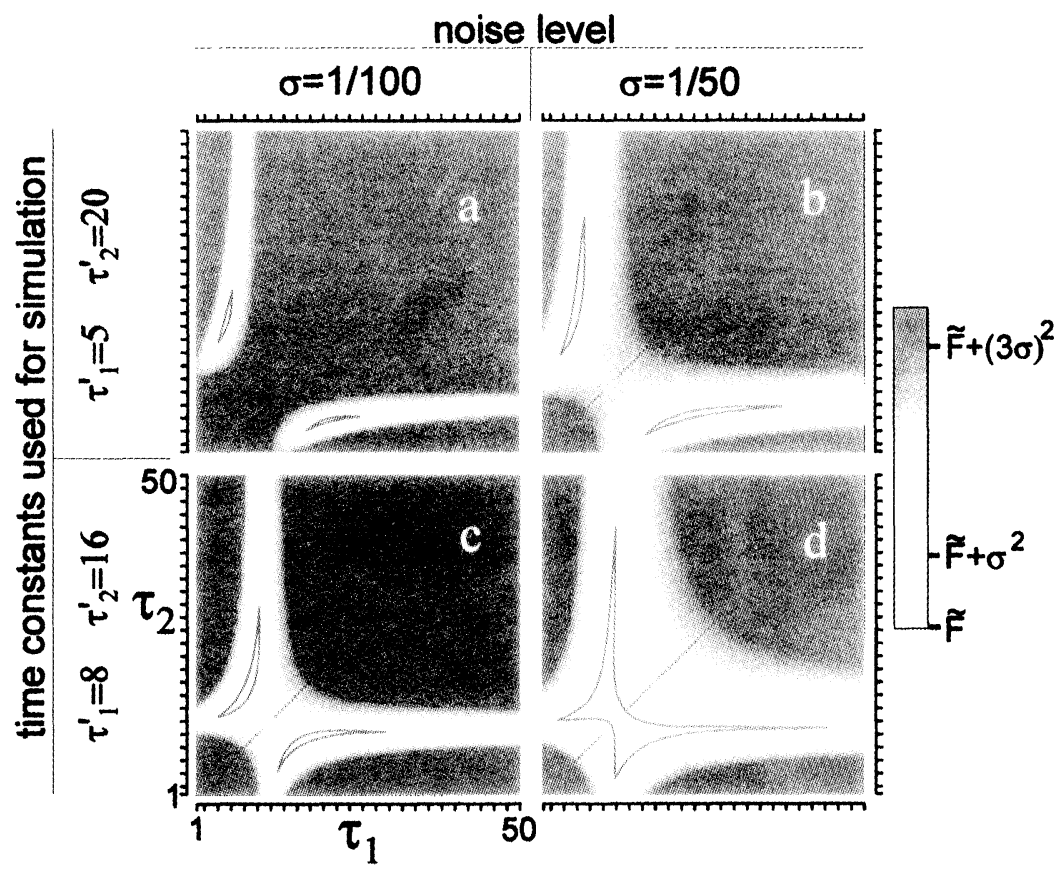

FIGURE 3 Density plots of the function $F\left(\tau_{1}, \tau_{2}\right)$ for simulated data. The blackness represents the value of $F\left(\tau_{1}, \tau_{2}\right)$. This function gives the error of estimation of the fitfunction $f\left(\tau_{1}, \tau_{2}\right)$. The white area represents the set of solutions $\mathcal{L}$ for which the criterion Eq. (6) holds approximately. The grey points show the function $F\left(\tau_{1}, \tau_{2}\right)$ in the surrounding area. The data points were obtained from a simulation of 50 equidistant base points for $t=[0,50]$ according to Eq. (7). The following coefficients were used for the simulation: (a) $A^{\prime}=0.5, \tau_{1}^{\prime}=5, \tau_{2}^{\prime}=20, \sigma^{-1}=100$; (b) $A^{\prime}=0.5, \tau_{1}^{\prime}=5, \tau_{2}^{\prime}=20$, $\sigma^{-1}=50 ;$ (c) $A^{\prime}=0.5, \tau_{1}^{\prime}=8, \tau_{2}^{\prime}=16, \sigma^{-1}=100 ;$ (d) $A^{\prime}=0.5, \tau_{1}^{\prime}=8, \tau_{2}^{\prime}=16$, $\sigma^{-1}=50$.

to the line $\tau_{1}=\tau_{2}$. The elements on this symmetry axis represent single-exponential functions. If one of the coefficients $\tau_{i}$ becomes large, the fit-function is also quasi-single exponential with an approximately constant offset. A different degree of broadening of the set of solutions can be observed if results obtained for simulated data of different $S / N$ are compared. As the relation $\tau_{1} / \tau_{2}$ comes closer to 1 , the correlation of $\tau_{i}$ increases. The shape of the set of solutions obtained for a level of significance $\alpha$ reflects this behaviour. Thus the geometry of the set of solutions is less parallel to the coordinate lines (compare a with $\mathrm{c}$ or $\mathrm{b}$ with $d$ of Fig. 3). It can be seen from Figure 3d that the existence of two different time constants can not be concluded without doubt in 
this case, because the set of solutions contains also symmetric solutions $\left(\tau_{1}=\tau_{2}\right)$.

The example shown in Figure 3 demonstrates the application of the scanning-fit method for the estimation of two-dimensional sets of solutions for the critical coefficients. In contrast to conventional fitalgorithms not only a point estimation was obtained, but detailed information about the possible vectors of fit-coefficients. They are given as the set of solution.

The calculation time for a scanning-fit depends linearly on the range and the resolution (sample rate) of the scanning of each of the critical parameters. Because it is the $S / N$ of the experimental data which determines the accuracy of the parameters a corresponding resolution (sample rate) exists which, however, does not make use to exceed. Thus, no fit-method can increase the numerical accuracy of the solution found for $\tilde{p}_{c}$ in general. Nevertheless, in contrast to conventional fit methods the scanning-fit guarantees that all solutions within the scanned domain (including the global minimum) are found.

\subsection{Global Scanning-fit}

If some coefficients of a series of measurements are known to be equal (global coefficients), this constraint can be used to improve the results of fit-algorithms. This kind of data analysis is named global fitanalysis. The introduced method of scanning-fit can be used effectively for this kind of data analysis. Different techniques are possible, most commonly the global coefficients are also the critical coefficients and a very graphic solution can be found. If we consider that the results of a series of experiments, $\varepsilon$ can be coupled, a global set of solutions can be obtained from the intersection of all solutions $\mathcal{L}_{\alpha}$ obtained for the single experiments.

$$
\hat{\mathcal{L}_{\alpha}}=\bigcap_{\varepsilon} \mathcal{L}_{\alpha}^{\varepsilon}
$$

This construction can be illustrated as the "optical multiplication" of the density plots obtained for the single experiments. (see Fig. 4).

A similar solution can be obtained if the global hyper-surface is calculated from the single hyper-surfaces and the scanning-fit method 


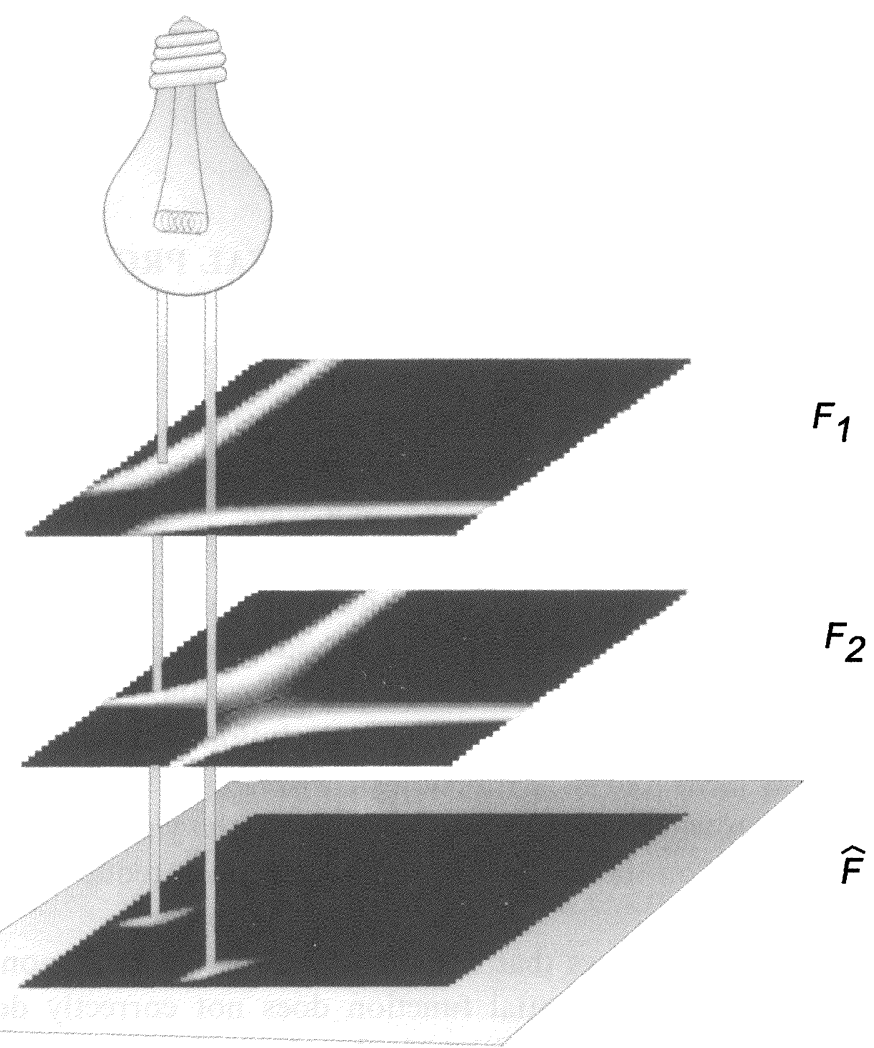

FIGURE 4 Cartoon of the optical multiplication of density plots obtained as graphic results of scanning-fit analysis of two experiments. The coupling of the single experiments increases the accuracy of $p_{c}$.

is applied to the global problem. The global chi-squared function $\hat{F}$ is the product of the single functions $F$.

$$
\begin{aligned}
\hat{F}\left(p_{c}, p_{r},\left\{y_{j},\left\{x_{1 . . m}\right\}_{j}\right\}_{\varepsilon}\right) & =\prod_{\varepsilon} F_{\varepsilon}\left(p_{c}, p_{r},\left\{y_{j},\left\{x_{1 . . m}\right\}_{j}\right\}\right) \\
& =\prod_{\varepsilon}\left[\frac{1}{a_{\varepsilon}} \sum_{j}\left(f\left(p_{c}, p_{r},\left\{x_{1 . . m}\right\}_{j}\right)-y_{j}\right)_{\varepsilon}^{2}\right]
\end{aligned}
$$

The application of the introduced method to this function of global coefficients $p_{c}$ and the variable remaining coefficients $p_{r, \varepsilon}$ yields the global set of solutions. All properties of the simple scanning-fit hold 
for the global scanning-fit. In contrast to conventional fit-algorithms the scanning-fit results of different experiments can be compared and combined effectively.

\section{APPLICATION TO PHOTOPHYSICAL PROCESSES}

\subsection{Monoexponential Relaxation of Pinacyanol}

Pinacyanol (1,1-diethyl-2,2 carbocyanin chloride) $\left(\lambda_{A b}=605 \mathrm{~nm}\right)$ is a polymethine dye which exhibits photoisomerization $[5,6]$ with an isomer absorption band on the long wave side at $635 \mathrm{~nm}$. The isomerization rate is solvent dependent. We have studied the transient absorption of pinacyanol in methanol with the aim to find out whether one or two decay processes are involved. Therefore, the dynamics of the light induced processes of excited pinacyanol were studied using pump-probe technique with fs-time resolution. The change in absorption was measured as a function of wavelength and delay time. The integration within a range of the wavelengths yields functions which depend only on the time. Thus the dimension is reduced to $m=1$ (see Eq. (2)).

The analysis shows that isomerization is performed monoexponentially and a biexponential function does not correctly describe the relaxation mechanism of the excited molecules. To test this behaviour, a biexponential function was used for the scanning-fit.

$$
\Delta A(t)=\frac{1}{1+\mathrm{e}^{-2 \cdot t / \tau_{p}}} \cdot\left(a_{1} \cdot \mathrm{e}^{-t / \tau_{1}}+a_{2} \cdot \mathrm{e}^{-t / \tau_{2}}+a_{3}\right)
$$

This fit function is an approximation for the convolution of the relaxation function and the function of the excitation pulse (see appendix). The amplitude $a_{3}$ reflects the long-lived photoisomer. The set of solution in Figure 6 includes symmetrical solutions $\left(\tau_{1}=\tau_{2}\right)$, where the quality of the fit function is not significantly lower than that of possible asymmetrical solutions. Previous experiments revealed also monoexponential behavior [7]. Our experiments yield the same result, however, due to the increased time resolution a biexponential behavior can be excluded with a high probability. It should be remarked, that the timescales of some ultrafast dynamics of the solvent (e.g., inertial solvation response in methanol [8]) are fast compared with 


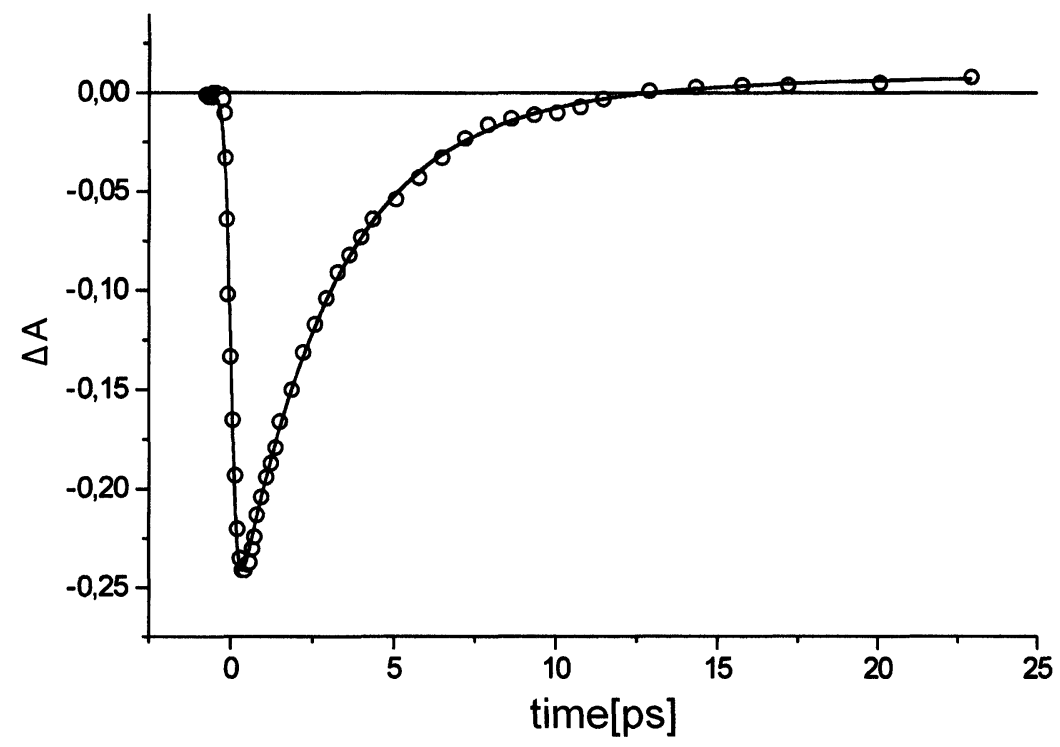

FIGURE 5 Transient absorption $\Delta A(t)$ of pinacyanol dissolved in methanol. The curve shows the induced fluorescence (negative sign) later on the photo isomer absorption (positive sign). The data points (o) are the result of an integration within the spectral range between $690 \mathrm{~nm}$ and $710 \mathrm{~nm}$. The fit function $(-) \Delta A(t)=1 /(1+$ $\left.\exp \left(-2 \cdot t / \tau_{p}\right)\right) \cdot\left(a \cdot \exp \left(-t / \tau_{1}+b \cdot \exp \left(-t / \tau_{2}\right)+c\right)\right.$ results $\tau_{1}=\tau_{2}=3 \mathrm{ps}, \tau_{p}=0.19 \mathrm{ps}$, $a+b=-0.287, c=0.003$. The excitation term is described in the appendix.

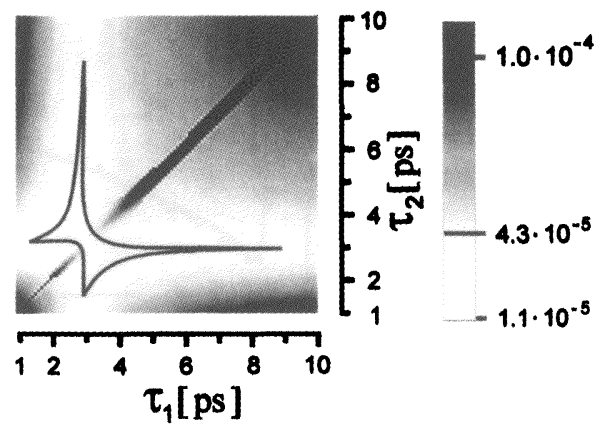

FIGURE 6 Density plots for the kinetics in the spectral range between $690 \mathrm{~nm}$ and $710 \mathrm{~nm}$ in Figure 5 which are obtained in a pump probe experiment with pinacyanol dissolved in methanol. The blackness (see gray scale) corresponds to the value of $F$ depending on $\tau_{1}$ and $\tau_{2}$.

the temporal resolution of this experiment. Additionally, transient absorption measurements are less sensitive to these processes, because cancellation of different response functions occur [9]. 
The monoexponential behavior allows the following conclusions on the relaxation process of pinacyanol solved in methanol:

- No long-lived electronically excited intermediate states are populated.

- Isomerization occurs either via one reaction pathway or through more having equal velocities.

As can be seen from the given example, the biexponetial scanning-fit clearly shows that a monoexponential function is sufficient to describe the experimental data. In this way the scanning-fit protects the user from the overinterpretation of the experimental data. This is in contrast to the solutions obtained from conventional fit methods. In the best case these methods yield the position of the global minimum leading to biexponential solutions $\left(\tau_{1} \neq \tau_{2}\right)$.

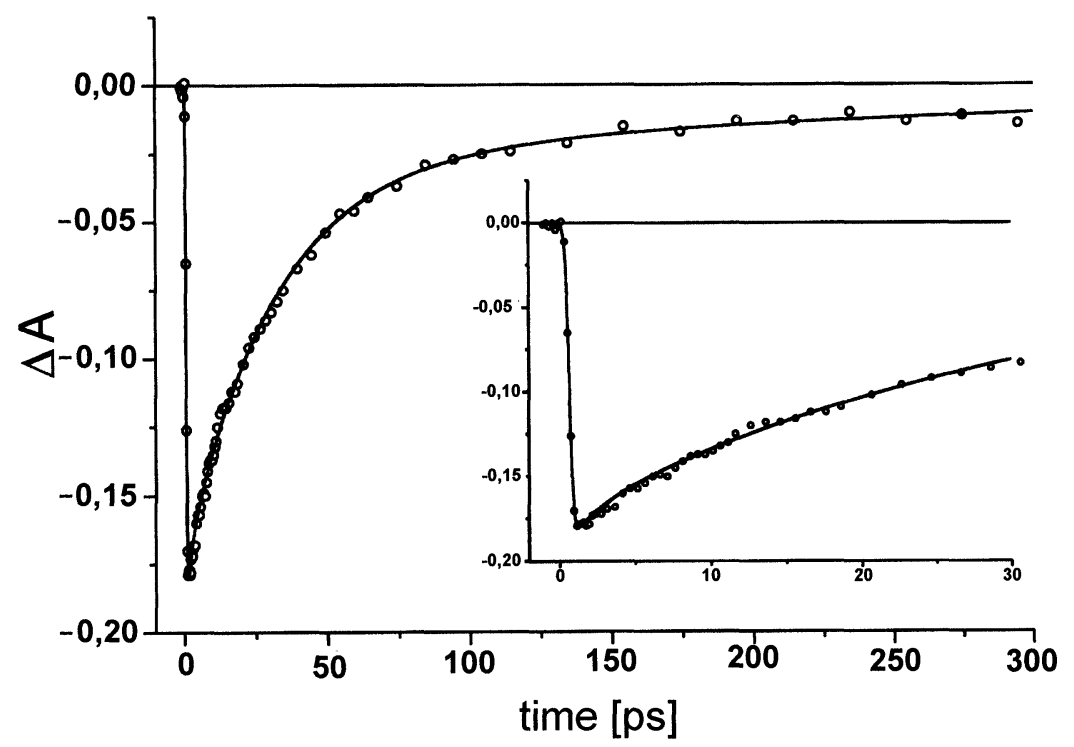

FIGURE 7 Transient absorption of Phycocyanobilin in methanol. The data (o) were obtained after integration within the spectral range of bleaching between $590 \mathrm{~nm}$ and $630 \mathrm{~nm}$. The negative $\Delta A$ reflects the bleaching after excitation at $610 \mathrm{~nm}$. The fit-function was $\Delta A(t)=1 /\left(1+\exp \left(-2 \cdot t / \tau_{P}\right)\right) \cdot\left(a \cdot \exp \left(-t / \tau_{1}\right)+b \cdot \exp \left(-t / \tau_{2}\right)+\right.$ $\left.c \cdot \exp \left(-t / \tau_{3}\right)\right) ; \tau_{1}=4 \mathrm{ps}, \tau_{2}=34 \mathrm{ps}, \tau_{3}=400 \mathrm{ps}, \tau_{P}=0.1 \mathrm{ps}, a=0.14, b=0.65, c=0.21$. The insert shows the data and the fit-function for short times. 


\subsection{Multi-exponential Relaxation of Phycocyanobilin}

From its chemical structure Phycocyanobilin is an open chain tetrapyrrole [10]. Different components (conformers, isomers, tautomers) of the molecule exist in the solvent methanol. Recently, the absorption spectra of different components were estimated from transient absorption measurements and the dynamics of excited components were studied [11]. Here we report some experimental data of these measurements to demonstrate the application of the scanning-fit method with a three-exponential fit-function.

The fit-function is of the type Eq. (4), supplemented with a factor which considers the temporal shape of the pump-pulse (for details see Appendix). The parameters $\tau_{i}$ are the critical coefficients of the fit question. In comparison to the examples discussed above the number of these critical parameters is higher. Typically, a global scanning-fit analysis should be performed if the kinetic parameters of the processes of phycocyanobilin are to be determined with high accuracy. In this paper we abstain from this procedure to focus on the demonstration of the scanning-fit method. We discuss the results of a non-global scanning-fit analysis of the data obtained after integration within the spectral range between $590 \mathrm{~nm}$ and $630 \mathrm{~nm}$.

The Figure 8 shows two-dimensional cuts through $F\left(\tau_{1}, \tau_{2}, \tau_{3}\right)$. For the visualization a level of significance $(\alpha)$ was used which corresponds to $\sigma^{-1}=500$ in the example of simulated data (Section 2.3). This value for $\alpha$ was obtained from the criterion 6 . The most probable
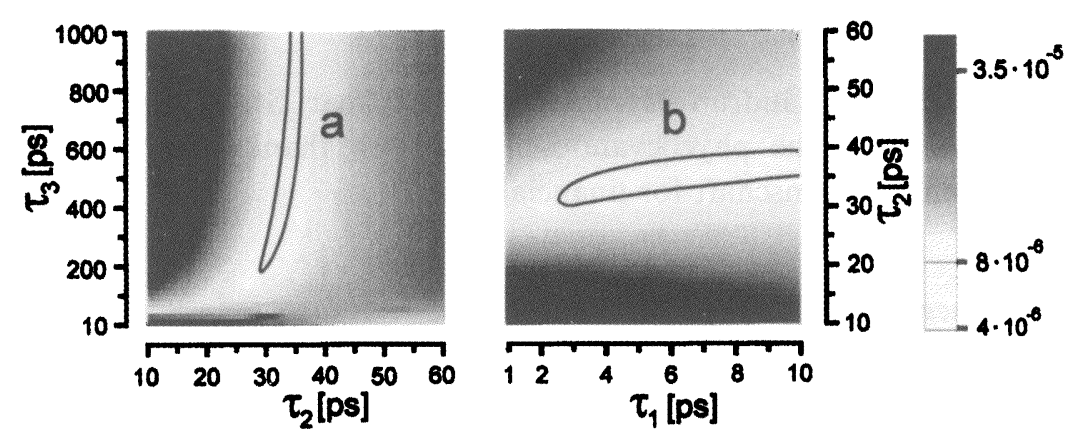

FIGURE 8 Density plots for data shown in Figure 7. The color represents the value of (a) $F\left(\tau_{1}, \tau_{3}\right)$ and (b) $F\left(\tau_{1}, \tau_{2}\right)$. The white area represents the set of solutions $\mathcal{L}$. 
solutions are in the ranges of $\tau_{1}=(3 . .8) \mathrm{ps}, \tau_{2}=(30 . .40) \mathrm{ps}$ and $\tau_{3}=(200 . .700) \mathrm{ps}$.

In contrast to other fit-methods which yield only a point estimation, the scanning-fit method gives realistic ranges of confidence. Thus the example shows that globalized data analysis methods are to be prefered in the case of multi-exponential data analysis.

\section{CONCIUSION}

The main emphasis of this article is the characterization of the scanning-fit method. Starting with the basics of all fit methods, we formulated the principle of the scanning-fit. Afterwards this method was applied to simulated data and the main features of the obtained results were discussed. Fs-spectroscopic data concerning the photo isomerization of the polymethine dye pinacyanol were analyzed to demonstrate the capacity of the scanning-fit method. As expected, the obtained result are in agreement with those of former studies [5-7, 12]. Nevertheless, the new data are of a higher significance, corresponding to the increase of the time resolution and $S / N$.

Furthermore, we give an example for the application of the new method in the case of more then two critical parameters. The obtained results show that global fit methods should be used for the multiexponential data analysis. The theory of the global scanning-fit is discussed as well in this article.

\section{Acknowledgements}

We thank Dr. Gudrun Hermann and Dietmar Strehlow for the preparation of the phycocyanobilin. We also thank the Deutsche Forschungsgemeinschaft for financial support.

\section{References}

[1] Herrmann, J. and Wilhelmi, B., Lasers for Ultrashort Light Pulses (AkademieVerlag, Berlin, 1984).

[2] Mukamel, S., Nonlinear Optical Spectroscopy (Academic Press, London, 1997).

[3] Appenroth, K., Bischoff, M., Gabrys, H., Stoeckel, J., Swartz, H., Walczak, T. and Winnefeld, K. (2000). J. Inorg. Biochem., 78, 235-242. 
[4] Stobrawa, G. and Bischoff, M., Demonstration Program for the Scanning-Fit. The demonstration program for the scanning-fit is free and may be downloaded from the download page http://www.physik.uni-jena.de/ scanning-fit.

[5] West, W., Pearce, S. and Grum, F. (1967). J. Phys. Chem., 71, 1316.

[6] Rentsch, S., Danielius, R. V. and Gadonas, R. A. (1981). Chem. Phys., 59, 9.

[7] Sundström, V. and Gillbro, T. (1981). Chem. Phys., 61, 258.

[8] Kumar, P. V. and Maroncelli, M. (1995). J. Chem. Phys., 103, 3038-3060.

[9] Joo, T., Jia, Y., Yu, J.-Y., Lang, M. J. and Fleming, G. R. (1996). J. Chem. Phys., 104, 6089-6108.

[10] Falk, H., The Chemistry of Linear Oligopyrroles and Bile Pigments (SpringerVerlag, 1989).

[11] Bischoff, M., Hermann, G., Rentsch, S., Strehlow, D., Winter, S. and Chosrowjan, H. (2000). J. Phys. Chem., 104, 1810-1816.

[12] Rentsch, S. and Wilhelmi, B. (1983). J. Mol. Structure, 114, 1.

\section{APPENDIX}

Concerning the instantaneously electronic excitation of a two-level system at $t=0$ the change of population $\Delta n(t)$ of the system can be described with the relaxation function $f(t)$. The convolution with the exciting Delta-pulse yields the product of the relaxation function with the Heavyside step function $\Theta(t)$.

$$
\Delta n(t)=\Theta(t) \cdot f(t)
$$

If a laser pulse with the temporal shape $I(t) \sim \operatorname{sech}^{2}\left(t / \tau_{P}\right)$ is used the excitation is not instantaneous. If all relaxation processes are slow compared with $\tau_{P}$ and if no saturation of the transition occurs the change of population $\Delta \tilde{n}(t)$ follows integral over the laser pulse

$$
\Delta \tilde{n}(t)=\int_{-\infty}^{t} \operatorname{sech}^{2}\left(\frac{\xi}{\tau_{P}}\right) d \xi \sim \frac{1}{1+\exp \left(-2 t / \tau_{P}\right)}
$$

The substitution of $\Theta(t)$ in Eq. (12) with Eq. (14) yields an approximation for the convolution of the relaxation function and excitation function:

$$
\Delta n(t) \approx \frac{1}{1+\exp \left(-2 t / \tau_{P}\right)} \cdot f(t)
$$

This approximation holds if the shortest time constants of the relaxation process is greater then $2 \cdot \tau_{\mathrm{P}}$. 